\title{
Design Framework of Collision Avoidance and Energy Efficient MAC for MWSN
}

\author{
Getsy S. Sara and Sridharan D.
}

\begin{abstract}
Mobile Wireless Sensor Network (MWSN) adds new design dimension to the existing MAC protocols due to the mobility of nodes. Collision probability and communication power consumption is increased in MWSN. In this paper, a hybrid MAC protocol which minimizes collision and achieves energy efficiency is proposed. Clustering of mobile nodes is done here and scheduled slots are provided by fusion nodes to each mobile sensor node during intra precinct communication. Contention based communication with strobed preamble is applied for inter precinct communication between gateway nodes. It avoids collision probability by applying RTS-CTS mechanism in tandem with data fragmentation. By simulating with OMNET++, the efficacy of this protocol can be evaluated.
\end{abstract}

Index Terms - Collision avoidance, energy efficiency, MAC protocol, mobile wireless sensor network.

\section{INTRODUCTION}

Mobile wireless sensor network (MWSN) is an emerging technology that has attracted much research attention in the recent years. It consists of large number of tiny low power, cheap sensor nodes that have sensing, data processing and communicating capabilities and are mobile as they are attached to vehicles, humans, animals, mobilizers etc. It offers various advantages than static wireless sensor networks such as dynamic network coverage, replacing failed routing nodes, data muling etc. [1]. Energy efficiency is a very important parameter as these nodes consist of small battery power. The network lifetime depends on energy efficiency and therefore reducing the energy consumption plays a very crucial role. Major energy consumption occurs during communication. Energy wastage occurs due to collision, overhearing, control packet overhead and idle listening. A good MAC protocol must help to reduce the energy consumption [2] and avoid collision from interfering nodes. Most of the traditional Mac protocols of WSN try to efficiently generate duty cycle without taking into consideration the reduction of data packet collisions. Retransmission of packet consumes more energy [3]. Therefore it becomes important to design a MAC protocol that reduces packet collisions and generates good duty cycle.

This paper is distributed as follows - Section II deals with

Manuscript received April 10, 2012; revised June 12, 2012. This work was supported by Junior Research Fellowship for Engineering and Technology under University Grants Commission, India.

Getsy S Sara is with Dept. of Electronics and Communication Engineering, CEG, Anna University, Chennai, India (phone: 0984110582; e-mail: getsysudhir@gmail.com).

Sridharan D is is currently working as an Associate Professor in the Dept. of Electronics \& Communication Engineering, CEG, Anna University, Chennai, India (e-mail: sridhar@annauniv.edu). prior works done in this area. Section III gives the design details of the Collision Avoidance Energy Efficient MAC protocol. Section IV discusses the simulation methodology of the proposed MAC protocol. Section V concludes this paper.

\section{RELATED WORK}

Energy efficient protocols minimize the energy consumption during network activities. The network lifetime of mobile sensor network is improved by applying energy aware routing and energy saving techniques. The radio energy consumption is of the same order of magnitude in reception, transmission and idle states while the power consumption drops of at least one order of magnitude in sleep state. Therefore the radio should be put to sleep whenever possible. There are three main techniques to reduce power consumption - duty cycling, data driven approaches and mobility [4].

S-MAC [5] and T- MAC [6] assign a schedule to sensor nodes for regulating sleep and wake up time [3]. The high complexity in the design incurred due to periodic synchronization among neighbor nodes makes it undesirable for event based applications. Collision probability is also high. B-MAC [7] is a carrier sense media access protocol for wireless sensor network that provides a flexible interface to obtain ultra low power operation, effective collision avoidance and high channel utilization. Overhearing issue is not solved here. A long preamble increases the power consumption of all nodes in the sender's transmission coverage [8].

The authors in [1] have designed a hybrid MAC protocol called MEMAC that informs the sensor nodes when to wake up and when to go to sleep to save energy. It is an adaptive mobility aware and energy efficient protocol. It dynamically adjusts the frame size to enable the protocol to effectively adapt itself to changes in mobility and traffic conditions. This avoids wasting slots by excluding the nodes which have no data to transmit from the TDMA schedule and to switch nodes to sleep mode when they are not included in the communication.

Romain Kuntz and Thomas Noel [9] have introduced MACHIAVEL, a new method to access the medium. It allows the mobile sensors to capture the channel even in networks with high contention, while guaranteeing the synchronization with their peers. It is a sampling protocol that guarantees a mobile node that its neighbors are synchronized when emitting data. It also reduces the delay to access the medium and hence avoids the mobile node to saturate its packet queue. For multi hop operation, the combination of medium borrowing with a priority queue reports data faster to the sink. Significant reduction in packet 
losses and end to end delay in dense network is achieved. The disadvantage is that this protocol is designed only for fixed sensor infrastructure.

Ananta Pandey and Jae Sung Lim [10] proposed EMPT an energy efficient MAC protocol in TDMA environment for multi hop broadcast with a trickle support. EMPT is based on clustering and slot assignment for every transmitting node. The sleeping time is distributive and since only cluster head transmits and all other nodes receive, there will be massive reduction in transmission.

\section{COLlision AvoidAnCE ENERGy EFFICIENT MAC}

Collision Avoidance Energy Efficient MAC protocol is a hybrid protocol which exploits the energy efficient characteristics of low duty cycled contention based protocol and efficient channelization characteristics of scheduled MAC protocols [11]. The entire sensor network area is assumed to be circumscribed into a big square and then divided into different square zones called precincts. Initially the sensor nodes in the precinct elect a fusion head randomly [12]. Each sensor node calculates its probability of becoming a fusion head $\mathrm{P}\left(\mathrm{F}_{\mathrm{i}}\right)$ based on node's mobility metric, transmission range and surplus energy [12], [13], [14], [15].

$$
\begin{aligned}
P\left(F_{i}\right) & =P\left(E_{i}\right) * P\left(F_{i} / E_{i}\right)+P\left(R_{i}\right) * P\left(F_{i} / R_{i}\right) \\
& +P\left(M_{i}\right) * P\left(F_{i} / M_{i}\right)
\end{aligned}
$$

where

$E_{i}$ - Event that the node $\mathrm{i}$ has residual energy greater than $E_{t h}$.

$R_{i}$ - Event that the node $\mathrm{i}$ has transmission range greater than $R_{t h}$.

$M_{i \text { - }}$ Event that the node i's mobility metric is lesser than $M_{t h}$.

$E_{t h}, R_{t h}, M_{t h}$ are the threshold values of surplus energy, transmission range and mobility respectively.

Surplus energy,

$$
E_{s}=E_{i}-\left[e_{s}+e_{g} * r_{i}+e_{r} * r_{j}+e_{i j} * r_{i}\right]
$$

Transmission Range,

$$
R_{\max }=[\lambda / 4 * \Pi] *\left[\sqrt{\left(P_{t} * G_{t} * G_{r} *\left(1-|\Gamma|^{2}\right)\right.} / P_{r}\right]
$$

Mobility Metric,

$$
M(t)=(1 / N) \sum_{i=0}^{N-1} M_{i}(t)
$$

where

$e_{r}$ - Power consumed for receiving one bit of data

$e_{t}$ - Power consumed for transmitting one bit of data to a neighboring node $\mathrm{j}$

$r \&, r_{j}-$ traffic generating rate at node $\mathrm{i} \&$ node $\mathrm{j}$

$\lambda$ - Operating wavelength

$P_{t}$ - power transmitted by the sensor
$P_{r}$ - receiver sensitivity

$G_{t}-$ Gain of transmitting antenna

$G_{r}$ - Gain of receiving antenna

$|\Gamma|^{2}$ - reflected power coefficient of receiving antenna.

$M_{i}(t)$ - relative movement of other nodes to node i

$\mathrm{N}$ - Number of sensor nodes in a precinct

$$
M_{i}(t)=\left(\frac{1}{N-1}\right) * \sum_{j=0}^{N-1}\left|d_{i j}^{\prime}(t)\right|
$$

$$
\begin{array}{|l|l|}
\hline \text { CS } & \text { Wake Up Message } \\
\hline
\end{array}
$$

\begin{tabular}{|l|l|l|l|l|}
\hline CS & RTS & CTS & DATA & ACK \\
\hline
\end{tabular}

Fig. 2. Frame structure of message slot

where

$d_{i j}(t)$ - distance between node $\mathrm{I}$ and node $\mathrm{j}$ at time $\mathrm{t}$

$d^{\prime} i j(t)$ - time derivative of $\operatorname{dij}(t)$

The node with the highest $P(F i)$ is chosen as the fusion head. Periodically, the $P\left(F_{i}\right)$ value is checked. If the $P\left(F_{i}\right)<$ $P_{t h}\left(F_{i}\right)$, then the fusion node sets its VID as 0 . A non fusion node which has the highest $P\left(F_{i}\right)$ at that time is chosen as next fusion head. $P_{t h}\left(F_{i}\right)$ is chosen as 0.5 . On becoming a precinct member, the VID is updated to any number between 1 and $\mathrm{N}$. The gateway nodes have at least one neighboring precinct node within its communication range.

\section{A. Initialization}

The fusion head organizes the time into fixed non overlapping frames [16]. Each frame consists of multiple Scheduled Wake up Slot (SWS) and multiple Message Slot (MS). Each SWS consists of a period for Carrier Sensing (CS) and Wake up message and each MS consists of CS and period for RTS/CTS/DATA/ ACK message as shown in fig.1 and fig.2. The same slots can be allocated to multiple precinct member nodes. The CS period is allocated to avoid collision when concurrent transmission is initiated by multiple senders. When a node enters a precinct, the initialization phase is performed. It broadcasts a preamble with its $\mathrm{P}\left(\mathrm{F}_{\mathrm{i}}\right)$. The preamble length is equal to the entire SWS size. On waking up, the fusion node sends the Register Message which consists of SWS No. (Obtained as VID mod M where VID is the virtual ID and M is the number of SWS slots). The node registers itself with the precinct by sending an acknowledgement and then goes to sleep.

\section{B. Intra Precinct Communication}

Each node wakes up at its own wake up slot to listen to the channel for Wake up message from the fusion head. If there is no message it forces itself to go back to sleep mode. When an event is detected by the sensor node, it randomly picks up a message slot and notifies the fusion node using the Event Message. The Event Message consists of the MS No. and the time of event occurrence. The RTS/CTS mechanism is used to reserve channel and avoid hidden terminal problem. When the fusion node receives more than one Event Message it turns on its radio in all the Message slots for possible data transmission. Fig. 3 shows this communication process. 


\section{Inter Precinct Communication}

The Inter Precinct communication occurs in an unscheduled manner. The fusion node multi casts the RREQ packet to the gateway node. If the clear channel is obtained during the CS period at the beginning of the MS, the fusion node sends the Route request (RREQ) immediately. The gateway node forwards the packet to the neighboring gateway nodes as shown in Fig.4. Gateway nodes communicate via unscheduled communication. A long preamble is multi casted by the gateway node.

The receiver gateway node wakes up and keeps itself awake till the RREQ is transmitted and a Route Reply (RREP) is sent back to the sender fusion node. The other neighboring gateway nodes set the Net Allocation Vector (NAV) timer to its duration. Then the receiving gateway node transmits the message to the neighboring fusion head using the Intra cluster communication. Once the source fusion node receives the new path via RREQ - RREP message, it forwards the data packets through this path.

\section{Data Forwarding by Gateway nodes}

The data forwarding utilizes the fragmentation mode specified by the IEEE 802.11 standard. A strobed preamble [17] is send by the gateway node. The strobed preamble consists of a series of short preamble packets, each containing the VID of receiver gateway node and has small pauses between each preamble packets. When a gateway node wakes up and receives a short preamble, it looks at the intended receiver VID in the packet. If the node is not the intended recipient, it goes back to sleep immediately; else it remains awake till for the subsequent data packets. The gateway node on receiving the short preamble sends an early acknowledgement during the short pauses.

The sender on receiving the acknowledgement stops sending the preamble and transmits the data packets. The data packet is fragmented [18]. The sender fusion node sends an RTS control packet to reserve the medium of the gateway node for data transmission using a random MS. This RTS packet also includes the duration for the transmission of the first fragment and the corresponding acknowledgement. A certain set of nodes receives this RTS and set their NAV according to the duration timer. The receiver gate way node answers with CTS, again including the duration of transmission up to acknowledgement. A different set of receivers gets this CTS message and sets their NAV. The sender fusion node now sends the first data frame, frag 1 after waiting for an Short Inter frame Spacing (SIFS)time. The frame frag $_{1}$ includes another duration value used to reserve the medium for the duration of transmission following the second fragment and acknowledgement. Several nodes may receive this message and set their NAV. Due to the mobility of nodes this will represent a different set of new nodes that are contending for the message slot. The receiver gateway node of frag1 answers directly after SIFS with the acknowledgement packet $\mathrm{ACK}_{1}$ including the reservation for the next transmission. This process continues till all data fragments are forwarded. Once this happens, then the nodes can compete again for the Message slot after waiting for a Data Inter frame Spacing (DIFS) time [18].

\section{Simulation EnVIRONMENT AND ANAlysis}

The OMNET++ simulator is used [19] for implementing this algorithm. A dense sensor network of 100 nodes is being simulated in a field with $5 * 25 \mathrm{~m}^{2}$ area. Each simulation last for 500 seconds. The random waypoint mobility model with a pause time of 30 seconds is used to simulate node movement. The mobile sensor node speed is set to be between $5 \mathrm{~m} / \mathrm{sec}$ and $20 \mathrm{~m} / \mathrm{sec}$. For radio power consumption setting, $\mathrm{E}_{\text {elec }}=50$ $\mathrm{nJ} / \mathrm{bit}, \mathrm{E}_{\mathrm{amp}}=100 \mathrm{pJ} / \mathrm{bit} / \mathrm{m} 2$ and a path loss exponent $\alpha=2$ is set. Each sensor node is assumed to have an initial energy of $5 \mathrm{~J}$.

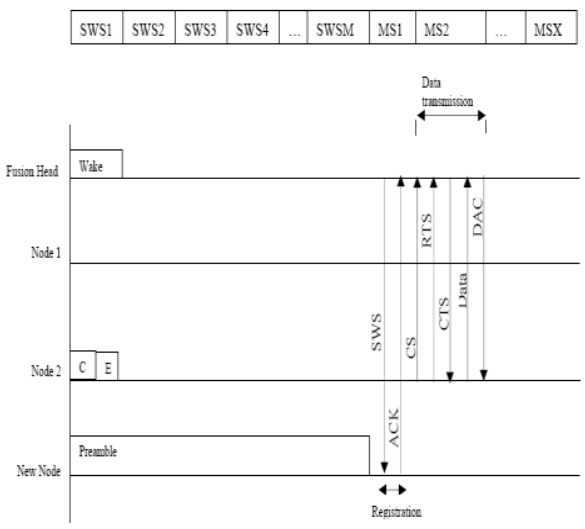

C- clear channel signaling, e- event message, dac- data acknowledgement Fig. 3. Intra precinct communication

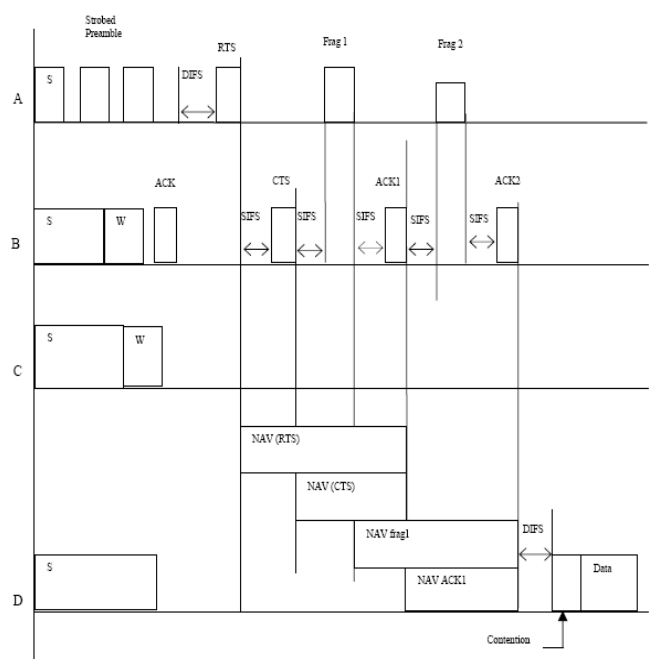

S - Sleep, W- wake, A - Sender gateway node, B- Receiver Gateway node, COverhearing, Gateway node, D- Contender Gateway Node

Fig. 4. Data forwarding by gateway nodes

The transmitting power and the receiving power of each sensor node are presumed to be $0.66 \mathrm{~W}$ and $0.395 \mathrm{~W}$ respectively. The $\mathrm{e}_{\mathrm{g}}$ and $\mathrm{e}_{\mathrm{s}}$ value is taken as $50 \mathrm{~mW}$ and 28.36 $\mathrm{mW}$ respectively and the radio range of a sensor node is $250 \mathrm{~m}$. Gt and $\mathrm{Gr}$ values are chosen as $1.2 \mathrm{dBi}$. $\alpha$ is assumed to be 0.3 and $\lambda$ is $900 \mathrm{MHz}$. The data is generated at a rate of $1 \mathrm{Kbps}$.

To evaluate the performance of the proposed protocol, two key performance metrics can be assessed; total energy consumption and packet delivery fraction Packet delivery fraction is defined as the ratio of data packets delivered to the destination to those generated by the source.

The proposed protocol is expected to guarantee reduction in energy wastage due to idle channel listening, overhearing and less collisions. The nodes do not collect the SWS 
information of its neighbors. Only the fusion node maintains it. This reduces communication overhead which can be very high in a dense or dynamic network [16]. During data transmission, the transmitter does not need to send the extended preamble, thus saving energy. The strobed preamble makes the non target gate nodes to go back to sleep immediately [17]. Even if nodes enter a precinct during communication, due to frag NAV and ack NAV, the nodes will not interfere in the communication. This prevents collision thereby reducing retransmission and hence saves energy [18].

\section{CONCLUSION}

This paper proposes a hybrid MAC protocol that saves energy consumption due to idle listening, collision avoidance and overhearing. The fusion head is chosen based on node's energy efficiency, mobility and transmission range. The goal of this protocol is to achieve reduction in energy consumption during communication without significantly degrading the network performance. During intra precinct communication, the time is organized into non overlapping frames and each precinct member is allocated a scheduled wake up slot for low power listening. Since we have focused on event based application, the message slot is provided randomly based on the need. The scheduled communication helps to reduce problems associated with interference among nodes. The clear channel signaling and the RTS-CTS mechanism employed here avoids hidden terminal problem. The data fragmentation done during inter precinct communication between gateway nodes help to reduce the collision further. The strobed preamble mechanism saves energy by allowing non target nodes to go back to sleep immediately. Therefore the proposed algorithm can guarantee better packet delivery ratio with less energy consumption and a longer network lifetime.

\section{REFERENCES}

[1] B. Yahya and J. B. Othman, "An adaptive mobility aware and energy efficient MAC protocol for wireless sensor networks," in the proceedings of 14th IEEE Symposium on Computers and Communications (ISCC 2009), July 5-8, Sousse, Tunisia, pp.15-21.

[2] B. Jang, J. B. Lim, and M. L. Sichitiu, "AS-MAC: an asynchronous scheduled MAC protocol for wireless sensor networks" in the proceedings of 5th IEEE International Conference on Mobile Ad Hoc and Sensor Systems, 2008, pp. $434-441$

[3] Z. Merhi, M. Elgamel, and M. Bayoumi, "EB-MAC: An event based medium access contol for wireless sensor networks," in the proceedings of IEEE International Conference on Pervasive Computing and Communications, 2009, percom, pp.1-6.

[4] G. Anastasi, M. Conti, M. Di Francesco, A. Passarella, "Energy Conservation in Wireless Sensor Networks: A Survey," In the Ad Hoc Networks, pp. 537-568.

[5] W. Ye, J. Heidemann, and D. Estrin, "Medium access control with coordinated adaptive sleeping for wireless sensor networks," IEEE/ACM Transactions on Networking, vol. 12, no. 3, pp. 493-506, June 2004

[6] T. V. Dam and K. Langendoen, "An adaptive energy efficient MAC protocol for wireless sensor networks," in SenSys' 03. ACM, 2003, Los Angeles, California, USA.
[7] J. Polastre, J. Hill, and D. Culler, "Versatile low power media access for wireless sensor networks," in SenSys'04. ACM, 2004, pp. 95-107.

[8] J. Bonny, "Investigating MAC power consumption in wireless sensor network," project report for the course of Self Organized Mobile Networks, Doctoral school of I and C School, winter semester 2004/2005, pp. 1-5.

[9] R. Kuntz and T. Noel, "Machiavel: Accessing the medium in mobile and dense WSN" in the proceedings of IEEE International Symposium on Personal, Indoor and Mobile Radio Communications, Sept13-16, 2009, Tokyo, pp. 1088-1092.

[10] A. Pandey and J. S. Lim, " Energy efficient MAC protocol for mobile wireless sensor networks," in the proceedings of the 9th international conference on Communications and information technologies, Incheon, Korea, 2009, pp. 629-630.

[11] H. S. Aghdasi and M. Abbaspour, "ET-MAC: an energy efficient and high throughput MAC protocol for wireless sensor networks" in the proceedings of 6th Annual communication Networks and Services Research Conference, 5-8 May 2008, Halifax, NS, pp. 526-532.

[12] G. S. Sara, R. Kalaiarasi, S. N. Pari, and D. Sridharan, " Energy Efficient Mobile Wireless Sensor Network Routing Protocol," in the Communications in Computer and Information Science, Recent Trends in Networks and Communications, Springer-Verlag Berlin Heidelberg , 2010, pp. $642-650$.

[13] Measure the Range Of Sensor Networks. [Online]. Available: www.mwrf.com/Article/ArticleID/19915/19915.html

[14] B. Jae, N. O. Song, L. E. Miller, "Standard Measure of Mobility for Evaluating Mobile Adhoc Network performance," in the IEICE Trans. Commun, vol. E86-B, no. 11, November 2003, pp. 3236-3243.

[15] C. L. Liu, D. P. Mohapatra, Elements of Discrete Mathematics, Third Edition, the McGraw- Hill Companies, pp: 89-92.

[16] H. Wang, X. Zhang, F. N. Abdesselam, and A. Khokhar, "Cross layer optimized MAC to support multihop QoSrouting for wireless sensor networks" in the IEEE Transactions on vechicular technology, vol. 59, no.5, June 2010, 2556-2563.

[17] M. Buttner, G. Yee, E. Anderson, and R. Han, "X-MAC: a short preamble MAC protocol for duty-cycled wireless sensor networks," Technical report CU-CS-1008-06, May 2006, pp: 1-11.

[18] J. Schiller, "Mobile Communications" Second Edition, Pearson Education Limited, pp: 219-220.

[19] OMNET++ Simulator. [Online]. Available: http:// www.omnetpp.org.

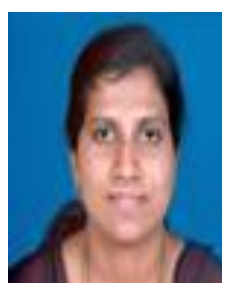

Getsy S. Sara received her B.E degree with distinction in Electronics \& Communication from Bharathiar University, India in 2004 and M.E degree with distinction in Digital Communication and Network Engineering from Anna University, India in 2006. Currently she is pursuing her Ph.D degree in the Faculty of Information \& Communication Engineering, Anna University Chennai, India. Her research interests include wireless ad hoc networking, sensor networks, energy efficient routing protocols and communication systems. She is an IEEE student member.

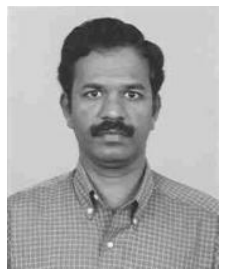

D. Sridharan received his B.Tech. degree and M.E.degree in Electronics Engineering from Madras Institute of Technology, Anna University in the years 1991 and 1993 respectively. He got his Ph.D degree in the Faculty of Information and Communication Engineering, Anna University in 2005. He is currently working as Associate Professor in the Department of Electronics and Communication Engineering, CEG Campus, Anna University, Chennai, India. He was awarded the Young Scientist Research Fellowship by SERC of Department of Science and Technology, Government of India. His present research interests include Internet Technology, Network Security, Distributed Computing and Wireless Sensor Networks. He is a life member of Institution of Electronics and Telecommunication Engineers (IETE), Indian Society for Technical Education (ISTE) and Computer Society of India (CSI). 